\title{
Controle do câncer de mama no estado de São Paulo: uma avaliação do rastreamento mamográfico
}

\section{Breast cancer control in São Paulo state: evaluation of mammogram screening}

Vívian Assis Fayer ${ }^{1}$ (D), Maximiliano Ribeiro Guerra', Mario Círio Nogueira², Camila Soares Lima Correa ${ }^{1}$, Lise Cristina Pereira Baltar Cury ${ }^{3}$, Maria Teresa Bustamante-Teixeira ${ }^{1}$

${ }^{1}$ Programa de Pós-graduação em Saúde Coletiva, Universidade Federal de Juiz de Fora (UFJF) - Juiz de Fora (MG), Brasil ${ }^{2}$ Departamento de Saúde Coletiva, Faculdade de Medicina, Universidade Federal de Juiz de Fora (UFJF) - Juiz de Fora (MG), Brasil ${ }^{3}$ Fundação Oncocentro de São Paulo (FOSP), Secretaria de Estado da Saúde de São Paulo - São Paulo (SP), Brasil

Como citar: Fayer VA, Guerra MR, Nogueira MC, Correa CSL, Cury LCPB, Bustamante-Teixeira MT. Controle do câncer de mama no estado de São Paulo: uma avaliação do rastreamento mamográfico. Cad Saúde Colet, 2020;28(1):140-152. https://doi.org/10.1590/1414-462X202028010322

\section{Resumo}

Introdução: O câncer de mama é o mais incidente, prevalente e com maior taxa de mortalidade entre as neoplasias malignas que acometem mulheres em todo o mundo, excluindo câncer de pele não melanoma. No Brasil, com exceção da região Norte, representa a mais frequente neoplasia maligna feminina. Objetivo: Estimar a cobertura de mamografias e analisar a qualidade e adequação às diretrizes técnicas nacionais do exame mamográfico em mulheres residentes na Região Metropolitana (RM) ou Interior do Estado (IE) de São Paulo, entre 2010 e 2012 Método: Estudo descritivo realizado a partir de dados do SISMAMA e SIA-SUS referentes ao período de 2010 a 2012. Resultados: Na RM paulista, não foi atingida a meta preconizada para a razão de mamografias em 2011 e 2012. Foi observado aumento do tempo de espera para acesso à mamografia diagnóstica e para a liberação deste resultado, e redução da capacidade de confirmação diagnóstica para as lesões suspeitas de malignidade no triênio. Já no IE, a meta preconizada para a razão de mamografias foi alcançada durante o período avaliado, com melhora da cobertura de biópsias em 2012. Em ambos (RM e IE), foi constatado elevado percentual de mamografia realizada fora da faixa etária preconizada (cerca de 35\%). Conclusão: Os resultados evidenciam a necessidade de adequações no rastreamento realizado no estado de São Paulo para que as ações de detecção precoce sejam efetivas. Destaca-se a importância da implantação de um rastreamento organizado e do aprimoramento do sistema de informação em saúde que possibilite o monitoramento e avaliação das ações, e contribua para o aperfeiçoamento da Política Nacional de Prevenção e Controle do Câncer de Mama.

Palavras-chave: neoplasias da mama; programas de rastreamento; mamografia; pesquisa sobre serviços de saúde; detecção precoce de câncer.

\footnotetext{
Abstract

Background: Breast cancer is the most incident, prevalent and the leading cause of female cancer death worldwide. In Brazil is the most commonly diagnosed female cancer, except in the North region. Objective: To evaluate coverage, quality and adequacy to the technical guidelines of breast cancer control

Trabalho realizado no Programa de Pós-graduação em Saúde Coletiva da Universidade Federal de Juiz de Fora (UFJF), situado no município de Juiz de Fora-MG.

Correspondência: Vívian Assis Fayer. E-mail: vivifayer@gmail.com

Fonte de financiamento: O presente trabalho foi realizado com apoio da Coordenação de Aperfeiçoamento de Pessoal de Nível Superior - Brasil (CAPES) - Código de Financiamento 001.

Conflito de interesses: Os autores declaram que não há conflito de interesses no presente artigo.

Recebido em: Jun. 19, 2018. Aprovado em: Maio 25, 2019
}

Este é um artigo publicado em acesso aberto (Open Access) sob a licença Creative Commons Attribution, que permite uso, distribuição e reprodução em qualquer meio, sem restrições desde que o trabalho original seja corretamente citado. 
actions indicators in women residing in the Metropolitan Region (MR) or in the Inland Towns of São Paulo, Brazil, between 2010 and 2012. Method: Descriptive study based on SISMAMA data from 2010 to 2012 was carried out. Results: MR did not reach the recommended target for the mammography ratio in 2011 and 2012, which presented a considerable percentage of mammogram beyond the recommended age range, increased the waiting time for access to diagnostic mammography and for obtain this result, and reduced the capacity diagnostic confirmation for lesions suspected of malignancy during the triennium. Inland towns achieved the recommended target for the mammography ratio over the period and improved biopsy coverage in 2012. Conclusion: The results highlighted requirement for adjustments in the screening actions in the state of São Paulo that allow the practice of early detection actions more effective, reinforce the relevance of the available Information Systems and their continuous improvement, as well as contribute to stimulate the evaluation practice and of the reviewing of the National Cancer Prevention and Control Policy.

Keywords: breast neoplasms; mass screening; mammography; health services research; early detection of cancer.

\section{INTRODUÇÃO}

O câncer de mama é o mais incidente, prevalente e com maior taxa de mortalidade entre as neoplasias que acometem mulheres em todo o mundo, excetuando-se os tumores de pele não melanoma ${ }^{1,2}$. No contexto global em 2012, sua estimativa era de aproximadamente 1,7 milhão de novos casos e 0,5 milhão de mortes causadas pela doença ${ }^{2}$. No Brasil, segundo o Instituto Nacional do Câncer (INCA), são estimados 56,33 casos em 2018. Com exceção da região Norte, representa a mais frequente neoplasia feminina, excluindo câncer de pele não melanoma ${ }^{3}$. A taxa de mortalidade pela doença tem aumentado nas cinco regiões do país nas últimas três décadas, entretanto, no início dos anos 1990 observou-se uma redução na mortalidade nas capitais da região Sul e Sudeste, concomitante com o aumento da mortalidade nos municípios do interior dos estados, especialmente do Norte e Nordeste ${ }^{4,5}$.

A detecção precoce da doença é a melhor estratégia para seu enfrentamento, e se dá por meio do diagnóstico precoce e do rastreamento ${ }^{1,6}$. Entende-se como diagnóstico precoce aquele realizado ainda em fase inicial da doença em mulheres sintomáticas, e rastreamento como a identificação de neoplasia em mulheres assintomáticas, geralmente realizado através do exame de mamografia ${ }^{6}$.

A estratégia de rastreamento organizado de base populacional tem sido amplamente recomendada e implementada de forma heterogênea em diversos países ${ }^{1,7}$. Caracteriza-se como um programa voltado para uma população-alvo previamente definida, convidada a participar das ações do rastreamento, com periodicidade predeterminada, com regularidade no monitoramento e na avaliação das ações desenvolvidas. Por outro lado, o rastreamento oportunístico é aquele que atende à demanda espontânea dos serviços de saúde, sem uma sistematização das ações de rastreamento e sem que ocorra um convite para população-alvo definida nas diretrizes adotadas ${ }^{6,8}$. O Brasil adota o modelo oportunístico, que tem se mostrado ineficiente por apresentar dificuldades políticas, logísticas, econômicas e socioculturais, além de ocasionar desigualdade no acesso e utilização das ações de rastreamento ${ }^{8-10}$.

Recentes evidências científicas indicam que o início do rastreamento mamográfico populacional antes dos 50 anos não produz redução na mortalidade ${ }^{6,11}$. No cenário nacional, as diretrizes para detecção precoce do câncer de mama assemelham-se às recomendações preconizadas pela Organização Mundial de Saúde (OMS), orientando a realização de mamografia em mulheres entre 50 e 69 anos, com periodicidade bienal| ${ }^{6,11-13}$. Contudo, algumas associações e sociedades de especialidades médicas têm recomendado o rastreamento anual entre 40 e 74 anos, fato que dificulta a adesão dos profissionais às recomendações do Ministério da Saúde e a unificação das práticas de rastreamento no país ${ }^{14}$.

Desde o final dos anos 1980, as ações para o controle do câncer de mama fazem parte das políticas públicas brasileiras de saúde, através de diretrizes voltadas à saúde integral da mulher. Como resultado da instituição, em 2005, da Política Nacional de Atenção Oncológica ${ }^{15}$, foi criado o Sistema de Informação do Controle do Câncer de Mama (SISMAMA) em 2009. Este é um subsistema do Sistema de Informação Ambulatorial do Sistema Único de Saúde (SIA-SUS), 
que contém informações sobre mamografias, exames citopatológicos e histopatológicos realizados no sistema público de saúde e que possui, dentre outras finalidades, o gerenciamento das ações de rastreamento e a disponibilização de dados que contribuam para o planejamento da oferta de serviços ${ }^{16,17}$. Atualmente as ações de controle do câncer de mama fazem parte do Plano de Ações Estratégicas para o Enfrentamento das Doenças Crônicas Não Transmissíveis (DCNT) no Brasil - 2011 a $2022^{18}$.

O monitoramento e avaliação das ações de rastreamento através dos indicadores de processo e resultado são fundamentais para que o controle da doença seja atingido. A produção desses indicadores segundo a faixa etária e periodicidade viabiliza a avaliação do direcionamento da oferta de exames para a população-alvo e contribui para a adequação da oferta de serviços ${ }^{19}$.

O objetivo do presente estudo foi estimar a cobertura de mamografias e analisar a qualidade e adequação às diretrizes nacionais do exame mamográfico no estado de São Paulo.

\section{MÉTODO}

Trata-se de estudo descritivo realizado a partir de dados do SISMAMA e SIA-SUS, referentes ao estado de São Paulo, no período compreendido entre 2010 e 2012.

O estado de São Paulo apresenta a maior densidade populacional entre os estados brasileiros ${ }^{20}$, com $51 \%$ da população composta por mulheres no ano de 2012 , dentre as quais 17,8\% encontravam-se na faixa dos 50-69 anos ${ }^{21}$, recomendada para o rastreamento do câncer de mama ${ }^{6,11}$. Ocupa o segundo lugar no ranking do Índice de Desenvolvimento Humano - IDH $(0,783)$ no Brasil, atrás apenas do Distrito Federal $(0,824)^{20}$. O estado possui 645 municípios, dentre os quais 39 compõem a região metropolitana de São Paulo ${ }^{22,23}$, regulamentada pela lei complementar estadual no 1.139 de 16 de julho de $2011^{24}$ com os seguintes municípios: Arujá, Barueri, Biritiba-Mirim, Caieiras, Cajamar, Carapicuíba, Cotia, Diadema, Embu, Embu-Guaçu, Ferraz de Vasconcelos, Francisco Morato, Franco da Rocha, Guararema, Guarulhos, Itapecerica da Serra, Itapevi, Itaquaquecetuba, Jandira, Juquitiba, Mairiporã, Mauá, Mogi das Cruzes, Osasco, Pirapora do Bom Jesus, Poá, Ribeirão Pires, Rio Grande da Serra, Salesópolis, Santa Isabel, Santana de Parnaíba, Santo André, São Bernardo do Campo, São Caetano do Sul, São Lourenço da Serra, São Paulo, Suzano, Taboão da Serra e Vargem Grande Paulista. Tais municípios compõem uma divisão administrativa da Secretaria de Saúde denominada Departamento Regional de Saúde da Grande São Paulo, que é o resultado da união de seis regiões de saúde (Grande ABC, Alto do Tietê, Franco da Rocha, Mananciais, Rota dos Bandeirantes, São Paulo), onde se concentram importantes centros de referência na assistência oncológica. Esta região metropolitana reúne cerca de $50 \%$ da população estadual, engloba relevantes atividades industriais, comerciais e financeiras, concentrando alto Produto Interno Bruto (PIB), que corresponde a cerca de $18 \%$ do total nacional ${ }^{23,25}$.

Neste estudo, os indicadores foram calculados tanto para o estado de São Paulo, quanto para região metropolitana (RM), com seus 39 municípios e interior do estado (IE), que reuniu os demais municípios.

Para estimar a cobertura, analisar a qualidade e adequação às diretrizes técnicas nacionais do exame mamográfico, foram calculados os seguintes indicadores:

- Cobertura do rastreamento mamográfico: Razão de mamografias em mulheres na faixa etária de 50 a 69 anos. Estimada pela razão entre o número de mamografias realizadas em mulheres na faixa etária de 50 a 69 anos e a metade da população feminina nessa faixa etária, visto que a periodicidade recomendada é bienal (utilizada como proxy da cobertura);

- Adequação às diretrizes técnicas nacionais ${ }^{26}$ : Proporções de mamografias de rastreamento por faixa etária; faixa etária de 50 a 69 anos com mamografia anterior ao ano considerado; faixa etária de 50 a 69 anos segundo o tempo (em anos) de realização da mamografia anterior; 
- Qualidade do exame mamográfico: Proporções de mamografias segundo indicação clínica por: faixa etária; categoria BI-RADS ${ }^{\circledR}$; com realização de exame em até 30 dias e com resultado em até 30 dias; Razão entre biópsias e mamografias com resultados BI-RADS $^{\circledast} 4$ e 5 (utilizada como proxy da cobertura de exames histopatológicos).

Foram também analisadas as características das lesões malignas por faixa etária, tamanho do tumor e presença de linfonodo axilar palpável.

Os dados foram extraídos do SISMAMA e SIA-SUS, disponíveis no sítio eletrônico do Departamento de Informática do Sistema Único de Saúde (Datasus). O SIA foi utilizado somente para o cálculo da razão de mamografia, visto que apresentava maior número de registros de exames quando comparado ao SISMAMA. O total de mamografias unilaterais registradas no SIA (procedimento: 0204030030) foi reduzido à metade, de forma a equivaler ao número de mulheres avaliadas, visto que neste sistema as mamografias são registradas de forma duplicada para cada mulher ${ }^{13,27}$. Os denominadores populacionais foram obtidos a partir do censo demográfico de 2010 e das estimativas populacionais para 2011 e 2012 desenvolvidas pelo Instituto Brasileiro de Geografia e Estatística (IBGE). O cálculo deste indicador para os anos de 2010 e 2011 foi adaptado para a recomendação bienal do exame, como recomendado para o ano de 2012. Desta forma foi possível compará-los às metas estabelecidas no Sistema de

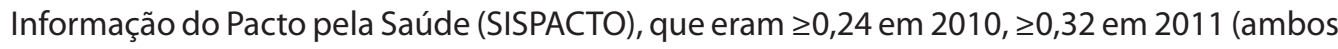
valores ajustados para periodicidade bienal) e $\geq 0,35$ em 2012 25,26.

O sistema de classificação Breast Imaging Reporting and Data System (BI-RADS ${ }^{\circledR}$ ), criado para padronizar os laudos mamográficos, e que na prática direciona a conduta clínica ${ }^{28}$, foi utilizado para subsidiar a avaliação da adequação do indicador "razão entre biópsias e mamografia alteradas" (BI-RADS ${ }^{\circledR} 4$ e 5), assim como na caracterização do perfil dos achados segundo faixa etária e indicação clínica.

Optou-se por avaliar o triênio de 2010 a 2012, visto que o SISMAMA foi implantado no segundo semestre de 2009 e, a partir de 2013, iniciou-se a migração das informações referentes às neoplasias da mama e do colo de útero para o Sistema de Informação do Câncer (SISCAN). Os dados foram extraídos entre outubro de 2017 e março de 2018. Informações mais detalhadas sobre o cálculo dos indicadores podem ser encontradas em Corrêa e colaboradores ${ }^{13}$.

O cálculo das razões e das proporções e a análise dos dados foram realizados no software Microsoft Excel 2013.

Este estudo integra projeto de pesquisa mais amplo, "Desigualdades de acesso às ações de controle de câncer através dos Sistemas de Informações de Saúde (DAAC-SIS)", aprovado pelo Comitê de Ética em Pesquisa da Universidade Federal de Juiz de Fora sob o parecer de no 1.431.916, e pelo Comitê de Ética em Pesquisa da Universidade de São Paulo sob o parecer de $n^{\circ} 2.209 .422$.

\section{RESULTADOS}

No estado de São Paulo, foram realizados 502.079 exames mamográficos em 2010, 621.101 em 2011 e 643.506 em 2012, sendo 45,7\% (2010), 43,5\% (2011) e 42,9\% (2012) na Região Metropolitana de São Paulo.

A razão de mamografias em mulheres na faixa etária de 50 a 69 anos no estado de São Paulo apresentou razões de 0,27, 0,33 e 0,34 para os anos de 2010, 2011 e 2012, respectivamente, enquanto as metas mínimas estabelecidas no Pacto pela Saúde para os mesmos anos foram de $0,24,0,32$ e 0,35 (metas adaptadas para a recomendação de realização bienal do exame). Para a RM, foi alcançada a meta preconizada em 2010 com razão de 0,26 e, nos anos seguintes, foram verificados valores inferiores aos preconizados, 0,30 em 2011 e 0,31 em 2012 (Figura 1). Já o IE apresentou valores de 0,27 (2010), 0,35 (2011) e 0,36 (2012), mantendo-se acima da meta nacional.

De acordo com o Censo 2010, entre 2010 e 2012 a população de mulheres da faixa etária de 40-49 anos era de, aproximadamente, 3 milhões; entre 50-59 anos, 2,3 milhões, e dos 60 aos 69 anos, 1,4 milhão. A maioria das mamografias de rastreamento foi realizada em mulheres da 


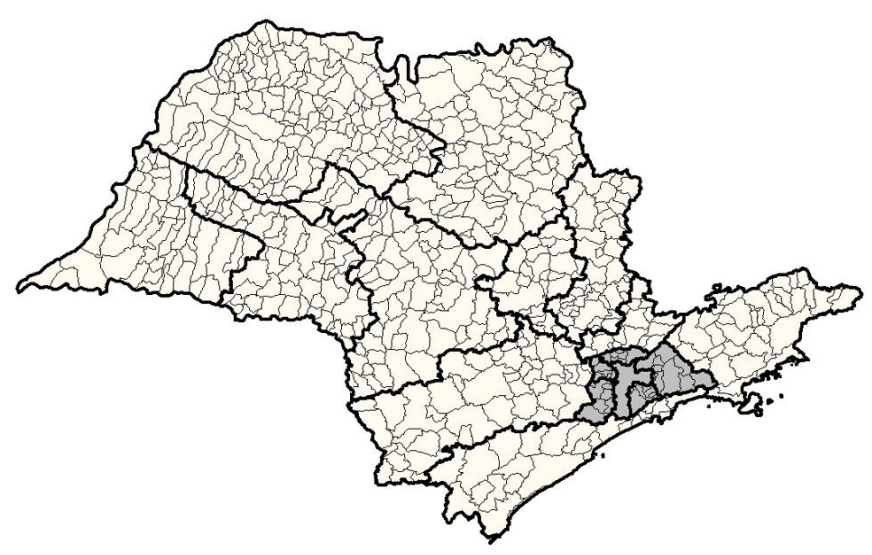

\section{Legenda:}

Região Metropolitana 2010: 0,26 2011: 0,30 2012: 0,31

$\begin{array}{ll}\text { Interior do estado } & \text { Meta naciona } \\ \text { 2010: } 0,27 & 2010: \geq 0,24 \\ \text { 2011: } 0,35 & 2011: \geq 0,32 \\ \text { 2012: } 0,36 & 2012: \geq 0,35\end{array}$

Figura 1. Razão de mamografia em mulheres na faixa etária de 50 a 69 anos para a região metropolitana e interior do estado de São Paulo, 2010-2012

faixa etária mais numerosa, de 40-49 anos, seguida pela faixa de 50-59 anos, que em conjunto correspondem a aproximadamente $70 \%$ dos exames realizados, independente do local e período avaliado (Tabela 1).

Com relação à proporção das mamografias de rastreamento na faixa etária de 50-69 anos, público-alvo do rastreamento, em torno de $85 \%$ das mulheres apresentavam mamografia anterior ao ano considerado, tanto na RM quanto no IE, e praticamente durante todo o triênio a periodicidade anual de realização da mamografia foi de $50 \%$ para a RM e de $45 \%$ para o IE. Cerca de $30 \%$ das mulheres rastreadas no estado de São Paulo realizaram mamografia com periodicidade bienal (Tabela 1).

A mamografia de rastreamento foi o principal tipo de indicação clínica na RM e no interior, com um percentual no estado de 93,3\% em 2010, 96,1\% em 2011 e 97,0\% em 2012.

Mesmo nas faixas etárias em que o exame não é recomendado $(<35,35-39,40-49$ anos, $\geq 70$ anos) foram registrados altos percentuais de rastreamento na $\mathrm{RM}$, sempre superiores a $91 \%$. No IE, verificou-se comportamento semelhante ao da RM, com exceção da faixa etária abaixo de 35 anos, que apresentou valores de 79,7\% (2010), 86,4\% (2011) e 85,8\% (2012). Destacam-se os percentuais inferiores a 10\% nas faixas etárias de 50-59 e 60-69 anos para as mamografias diagnósticas tanto na RM quanto no interior no período avaliado.

As proporções de mamografias de rastreamento com realização em até 30 dias e com resultado em até 30 dias se mantiveram similares na RM e IE entre os anos do triênio avaliado, apresentando proporções superiores às encontradas para as mamografias diagnósticas. Em contrapartida, os percentuais das mamografias diagnósticas sofreram redução entre $2010 \mathrm{e}$ 2012, de forma mais marcante na RM, principalmente nos resultados em até 30 dias (Tabela 2).

No estado de São Paulo, a distribuição proporcional de BI-RADS ${ }^{\circledR}$ segundo a indicação clínica apresentou os maiores percentuais nas categorias BI-RADS ${ }^{\circledR} 1$ (sem achado) e BI-RADS ${ }^{\circledR} 2$ (achados benignos) em ambas as indicações, rastreamento ou diagnóstica. A categoria BI-RADS $^{\circledR} 1$ exibiu comportamento decrescente e BI-RADS ${ }^{\circledR} 2$ crescente em relação à idade, independente da indicação. As categorias BI-RADS ${ }^{\circledR} 4$ (achados suspeitos de malignidade) e BI-RADS ${ }^{\circledR} 5$ (achados altamente sugestivos de malignidade) apresentaram percentuais mais expressivos nas mamografias diagnósticas, quando comparados aos de rastreamento, e aumentaram com a idade. As mamografias de rastreamento mantiveram um percentual em torno de $10 \%$ de exames inconclusivos (BI-RADS ${ }^{\circledR} 0$ ) entre os anos considerados, enquanto as mamografias diagnósticas sofreram redução entre 2010 e 2012, alcançando 4,5\% para todas as faixas etárias no final do triênio (Tabela 3). 
Tabela 1. Proporção de mamografias de rastreamento segundo faixa etária e de mulheres na faixa etária de 50 a 69 anos segundo o tempo (em anos) de realização. São Paulo - SP, 2010-2012

\begin{tabular}{|c|c|c|c|c|}
\hline \multicolumn{5}{|c|}{ MAMOGRAFIAS DE RASTREAMENTO (\%) } \\
\hline \multirow[t]{2}{*}{ Ano } & & $\begin{array}{c}\text { Região } \\
\text { Metropolitana }\end{array}$ & Interior & Estado SP \\
\hline & \multicolumn{4}{|c|}{ Faixa etária } \\
\hline \multirow{6}{*}{2010} & $<35$ & 1,3 & 1,0 & 1,1 \\
\hline & $35-39$ & 5,2 & 4,4 & 4,7 \\
\hline & $40-49$ & 36,0 & 36,3 & 36,2 \\
\hline & $50-59$ & 33,3 & 34,4 & 34,0 \\
\hline & $60-69$ & 17,2 & 17,8 & 17,6 \\
\hline & $\geq 70$ & 7,1 & 6,1 & 6,5 \\
\hline \multirow{6}{*}{2011} & $<35$ & 1,2 & 0,9 & 1,0 \\
\hline & $35-39$ & 4,8 & 3,8 & 4,2 \\
\hline & $40-49$ & 34,9 & 36,2 & 35,6 \\
\hline & $50-59$ & 33,4 & 34,6 & 34,1 \\
\hline & $60-69$ & 18,4 & 18,5 & 18,5 \\
\hline & $\geq 70$ & 7,4 & 6,1 & 6,6 \\
\hline \multirow{6}{*}{2012} & $<35$ & 1,0 & 0,8 & 0,9 \\
\hline & $35-39$ & 4,8 & 3,5 & 4,0 \\
\hline & $40-49$ & 35,1 & 36,5 & 35,9 \\
\hline & $50-59$ & 33,1 & 34,2 & 33,8 \\
\hline & $60-69$ & 18,8 & 18,8 & 18,8 \\
\hline & $\geq 70$ & 7,2 & 6,2 & 6,6 \\
\hline
\end{tabular}

Faixa etária de 50-69 anos segundo o tempo (em anos) de realização de mamografia anterior *

\begin{tabular}{|c|c|c|c|c|}
\hline & Periodicidade & & & \\
\hline \multirow{3}{*}{2010} & Até 1 ano & 52,9 & 49,8 & 50,8 \\
\hline & 2 anos & 31,2 & 31,7 & 31,5 \\
\hline & $\geq 3$ ou+ & 15,9 & 18,6 & 17,7 \\
\hline \multirow{3}{*}{2011} & Até 1 ano & 51,9 & 44,8 & 47,7 \\
\hline & 2 anos & 30,9 & 34,1 & 32,8 \\
\hline & $\geq 3$ out & 17,2 & 21,1 & 19,5 \\
\hline \multirow{3}{*}{2012} & Até 1 ano & 48,6 & 45,6 & 46,8 \\
\hline & 2 anos & 32,5 & 33,1 & 32,9 \\
\hline & $\geq 3$ out & 18,9 & 21,3 & 20,3 \\
\hline
\end{tabular}

*Lapso de tempo (em anos) compreendido pelo período entre o último registro de mamografia e a mamografia realizada em 2010, 2011 ou 2012 
Tabela 2. Proporção de mamografias com realização e resultado em até 30 dias segundo indicação clínica do exame por ano, na região metropolitana, interior e estado de São Paulo - SP, 2010-2012

\begin{tabular}{cccccccc} 
& \multicolumn{5}{c}{ Mamografia diagnóstica (\%) } & \multicolumn{3}{c}{ Mamografia rastreamento (\%) } \\
\hline \multirow{2}{*}{ Tempo } & Ano & $\begin{array}{c}\text { Região } \\
\text { Metropolitana }\end{array}$ & Interior & Estado & $\begin{array}{c}\text { Região } \\
\text { Metropolitana }\end{array}$ & Interior & Estado \\
\hline \multirow{2}{*}{$\begin{array}{l}\text { Realização em } \\
\text { até 30 dias }\end{array}$} & 2010 & 75.0 & 68.0 & 71.3 & 72.3 & 64.0 & 67.3 \\
\cline { 2 - 8 } & 2011 & 58.1 & 64.2 & 61.3 & 72.1 & 66.5 & 68.9 \\
\cline { 2 - 8 } & 2012 & 63.7 & 56.8 & 60.9 & 72.0 & 68.5 & 69.9 \\
\hline \multirow{2}{*}{$\begin{array}{l}2010 \\
\text { até 30 dias }\end{array}$} & 68.0 & 57.3 & 62.4 & 61.4 & 54.5 & 57.2 \\
\cline { 2 - 8 } & 2011 & 53.8 & 56.6 & 55.3 & 57.6 & 55.6 & 56.5 \\
\hline
\end{tabular}

Tabela 3. Distribuição proporcional de mamografias pela categoria BI-RADS segundo indicação clínica das mamografias, por ano e faixa etária, no estado de São Paulo - SP, 2010-2012

\begin{tabular}{|c|c|c|c|c|c|c|c|c|c|c|c|}
\hline \multirow[b]{2}{*}{ BI-RADS } & \multirow[b]{2}{*}{ Ano } & \multicolumn{4}{|c|}{ Mamografia diagnóstica (\%) } & \multicolumn{6}{|c|}{ Mamografia rastreamento (\%) } \\
\hline & & $40-49$ & $50-59$ & $60-69$ & $\geq 70$ & $\begin{array}{l}\text { Todas } \\
\text { faixas }\end{array}$ & $40-49$ & $50-59$ & $60-69$ & $\geq 70$ & $\begin{array}{l}\text { Todas } \\
\text { faixas }\end{array}$ \\
\hline \multirow{3}{*}{$\begin{array}{l}\text { BO } \\
\text { Inconclusivo }\end{array}$} & 2010 & 11.0 & 9.4 & 8.0 & 8.8 & 10.0 & 12.6 & 11.9 & 10.8 & 10.0 & 11.9 \\
\hline & 2011 & 7.9 & 6.8 & 6.1 & 6.2 & 7.3 & 11.3 & 10.8 & 9.9 & 9.3 & 10.7 \\
\hline & 2012 & 4.6 & 4.0 & 3.4 & 3.9 & 4.5 & 11.2 & 10.6 & 9.8 & 9.5 & 10.6 \\
\hline \multirow{3}{*}{$\begin{array}{l}\text { B1 } \\
\text { Sem achados }\end{array}$} & 2010 & 37.6 & 29.4 & 22.4 & 16.2 & 31.5 & 48.5 & 38.3 & 27.2 & 15.6 & 39.5 \\
\hline & 2011 & 35.0 & 26.8 & 20.6 & 14.9 & 28.8 & 46.2 & 36.6 & 25.9 & 15.0 & 37.4 \\
\hline & 2012 & 36.5 & 24.6 & 16.0 & 10.0 & 27.7 & 44.1 & 34.7 & 24.9 & 14.9 & 35.6 \\
\hline \multirow{3}{*}{$\begin{array}{l}\text { B2 } \\
\text { Achados } \\
\text { benignos }\end{array}$} & 2010 & 39.8 & 48.0 & 54.2 & 60.1 & 45.5 & 35.5 & 45.5 & 57.2 & 68.4 & 44.5 \\
\hline & 2011 & 38.9 & 46.1 & 52.7 & 60.4 & 45.1 & 39.4 & 48.7 & 60.0 & 70.3 & 48.1 \\
\hline & 2012 & 39.7 & 49.8 & 57.9 & 66.4 & 48.0 & 41.6 & 50.7 & 61.0 & 70.2 & 50.0 \\
\hline \multirow{3}{*}{$\begin{array}{l}\text { B3 } \\
\text { Achados } \\
\text { provavelmente } \\
\text { benignos }\end{array}$} & 2010 & 7.1 & 8.4 & 8.7 & 6.7 & 7.5 & 2.3 & 2.9 & 3.1 & 3.3 & 2.7 \\
\hline & 2011 & 12.2 & 13.1 & 12.3 & 8.6 & 11.5 & 1.9 & 2.5 & 2.6 & 3.0 & 2.3 \\
\hline & 2012 & 14.1 & 15.4 & 15.6 & 9.8 & 13.5 & 2.1 & 2.7 & 2.8 & 2.9 & 2.5 \\
\hline \multirow{3}{*}{$\begin{array}{l}\text { B4 } \\
\text { Achados } \\
\text { suspeitos de } \\
\text { malignidade }\end{array}$} & 2010 & 3.5 & 4.2 & 5.0 & 5.3 & 4.0 & 1.1 & 1.3 & 1.5 & 2.2 & 1.3 \\
\hline & 2011 & 4.9 & 5.7 & 6.2 & 6.5 & 5.5 & 1.1 & 1.2 & 1.4 & 2.0 & 1.3 \\
\hline & 2012 & 4.2 & 4.9 & 5.0 & 7.3 & 4.8 & 1.0 & 1.2 & 1.3 & 2.0 & 1.2 \\
\hline \multirow{3}{*}{$\begin{array}{l}\text { B5 } \\
\text { Achados } \\
\text { altamente } \\
\text { sugestivos de } \\
\text { malignidade }\end{array}$} & 2010 & 0.2 & 0.4 & 0.5 & 1.5 & 0.5 & 0.1 & 0.1 & 0.2 & 0.5 & 0.2 \\
\hline & 2011 & 0.5 & 0.8 & 1.1 & 2.1 & 0.9 & 0.1 & 0.1 & 0.2 & 0.4 & 0.1 \\
\hline & 2012 & 0.4 & 0.6 & 1.1 & 1.3 & 0.7 & 0.1 & 0.1 & 0.2 & 0.4 & 0.1 \\
\hline \multirow{3}{*}{$\begin{array}{l}\text { B6 } \\
\text { Biópsia } \\
\text { prévia com } \\
\text { malignidade } \\
\text { comprovada }\end{array}$} & 2010 & 0.9 & 1.1 & 1.2 & 1.5 & 1.1 & $x$ & $x$ & $x$ & $x$ & $x$ \\
\hline & 2011 & 0.7 & 0.8 & 0.9 & 1.4 & 0.9 & $x$ & $x$ & $x$ & $x$ & $x$ \\
\hline & 2012 & 0.5 & 0.7 & 1.0 & 1.3 & 0.8 & $x$ & $x$ & $x$ & $x$ & $x$ \\
\hline
\end{tabular}


A razão entre biópsias e mamografias com resultados BI-RADS ${ }^{\circledR} 4$ e 5 na RM apresentou valores, em 2010, que oscilavam entre 0,8 e 1,0 para as faixas etárias avaliadas, sendo superiores aos valores encontrados no IE. Para o triênio, o indicador se manteve estável no interior do estado e assumiu padrão decrescente na RM, oscilando entre 0,4 e 0,6, chegando a apresentar percentuais inferiores aos do IE, no ano de 2012.

Em relação ao número de biópsias da RM, foi observado déficit de aproximadamente $30 \%$ em 2011, quando comparada à produção de 2010, e em torno de $20 \%$ em 2012, quando comparada à de 2010 (ano de maior produção). O número de mamografias com resultado BI-RADS $^{\circledast} 4$ e 5 cresceu em ambas regiões no triênio, de forma mais marcante na RM, alcançando 48\% de aumento em relação à produção de 2012 e à de 2010 (Tabela 4).

No período avaliado, foram diagnosticados no estado de São Paulo 3.379 (2010), 3.815 (2011) e 4.435 (2012) casos de câncer de mama em mulheres, sendo a maioria das lesões detectadas por exame clínico ( $55 \%, 61 \%$ e $52 \%$, respectivamente), seguida pela detecção por imagem (38\%, 35\% e 43\%, respectivamente). As faixas etárias que concentraram os maiores percentuais de lesões malignas foram 50-69 anos, 40-49 anos e acima de 70 anos com média no triênio de, respectivamente, $48 \%, 23 \%$ e $19 \%$.

Quando considerada somente a faixa etária alvo do rastreamento, 50 a 69 anos, foram realizados 1.593 (2010), 1.879 (2011) e 2.399 (2012) diagnósticos de câncer de mama. Os percentuais de detecção por exame clínico foram de 52\% (2010), 58\% (2011) e 49\% (2012), enquanto os percentuais por exame de imagem foram 42\% (2010), 38\% (2011) e 47\% (2012). Entre aquelas mulheres que tiveram a lesão detectada por exame clínico, foram observados $21 \%$ de tumores menores que $2 \mathrm{~cm}, 48 \%$ com tumores de 2 a $5 \mathrm{~cm}$ e $20 \%$ com tumores maiores que $5 \mathrm{~cm}$, assim como presença de linfonodos axilares palpáveis em $44 \%$ das mulheres com neoplasia da mama no triênio. Já as lesões detectadas por imagem apresentaram, em média, tamanhos de tumores menores que $2 \mathrm{~cm}$ em $51 \%$, lesões de 2 a $5 \mathrm{~cm}$ em $26 \%$ e lesões com mais de $5 \mathrm{~cm}$ em 8\%, com ausência de linfonodos axilares palpáveis em 83\% entre 2010 e 2012.

Tabela 4. Razão entre biópsia e mamografias com resultados BI-RADS ${ }^{\circledR} 4$ (suspeito de malignidade) e 5 (altamente sugestivo de malignidade), por faixa etária, na região metropolitana e interior do estado de São Paulo, 2010-2012

\begin{tabular}{|c|c|c|c|c|c|c|c|c|c|c|c|}
\hline & & Região & Metrop & olitana & & & Interior & & & & \\
\hline Indicador & Ano & $40-49$ & $50-59$ & $60-69$ & $\geq 70$ & $\begin{array}{l}\text { Todas } \\
\text { faixas }\end{array}$ & $40-49$ & $50-59$ & $60-69$ & $\geq 70$ & $\begin{array}{l}\text { Todas } \\
\text { faixas }\end{array}$ \\
\hline \multirow{3}{*}{$\begin{array}{l}\text { Número de } \\
\text { biópsias }\end{array}$} & 2010 & 1433 & 1622 & 1156 & 825 & 6110 & 1247 & 997 & 745 & 503 & 4297 \\
\hline & 2011 & 1102 & 1128 & 804 & 513 & 4484 & 1467 & 1474 & 935 & 648 & 5660 \\
\hline & 2012 & 1192 & 1294 & 856 & 653 & 5181 & 1576 & 1481 & 1024 & 701 & 6102 \\
\hline \multirow{3}{*}{$\begin{array}{l}\text { Número de } \\
\text { mamografias } \\
\text { com resultado } \\
\text { Birads }{ }^{\circledR} 4 \text { e } 5\end{array}$} & 2010 & 1674 & 2130 & 1235 & 813 & 6196 & 2175 & 2350 & 1467 & 795 & 7161 \\
\hline & 2011 & 2453 & 2893 & 1958 & 1176 & 8952 & 2759 & 2979 & 1778 & 918 & 8845 \\
\hline & 2012 & 2616 & 3007 & 1934 & 1120 & 9180 & 2301 & 2596 & 1750 & 1014 & 8031 \\
\hline \multirow{3}{*}{$\begin{array}{l}\text { Razão entre } \\
\text { biópsias e } \\
\text { resultados } \\
\text { Birads } 4 \text { e } 5\end{array}$} & 2010 & 0.9 & 0.8 & 0.9 & 1.0 & 1.0 & 0.6 & 0.4 & 0.5 & 0.6 & 0.6 \\
\hline & 2011 & 0.4 & 0.4 & 0.4 & 0.4 & 0.5 & 0.5 & 0.5 & 0.5 & 0.7 & 0.6 \\
\hline & 2012 & 0.5 & 0.4 & 0.6 & 0.6 & 0.6 & 0.7 & 0.6 & 0.6 & 0.7 & 0.8 \\
\hline
\end{tabular}

\section{DISCUSSÃO}

A região metropolitana de São Paulo não atingiu a meta preconizada para a razão de mamografias em 2011 e 2012, exibindo considerável percentual dos exames fora da faixa etária preconizada (35\%, em média, no triênio), aumento no tempo de espera para acesso à mamografia diagnóstica e para a liberação deste resultado, e redução da capacidade de 
confirmação diagnóstica para as lesões suspeitas de malignidade. Já o interior do estado atingiu a meta durante o período avaliado, entretanto, com $36 \%$ de exames realizados fora da faixa etária preconizada. Obteve ainda aumento na oferta de biópsias e, em relação aos demais achados, exibiu comportamento semelhante à RM, todavia com percentuais inferiores.

Em ambas regiões, as mulheres precisaram esperar mais tempo para ter acesso à mamografia diagnóstica e ao resultado do exame. Quando comparamos os resultados de 2011 e 2012 com os valores apresentados em 2010, ano de melhor desempenho, observamos que especialmente na RM a prioridade da realização e liberação do resultado da mamografia diagnóstica não foi respeitada. No que se refere à oferta de exames histopatológicos, houve redução de 50\% em 2011 e 40\% em 2012 na RM e aumento de 20\% em 2012 no IE. Dados de 2013 e 2014 do Plano de Ação Regional de Prevenção e Controle do Câncer da Região Rota do Bandeirantes ${ }^{29}$, que inclui 7 cidades da RM (Barueri, Carapicuíba, Itapevi, Jandira, Osasco, Pirapora do Bom Jesus, Santana de Parnaíba), relatam a existência de déficit nos procedimentos de mamografias e de confirmação diagnóstica (histopatológico), cenário que possivelmente já existia no período de 2010 a 2012, que pode ter contribuído para justificar as diferenças encontradas entre a RM e o IE.

As razões de mamografia na RM de São Paulo em 2011 e 2012 apresentaram valores abaixo das metas nacionais preconizadas no Pacto pela Saúde ${ }^{18}$, exibindo valores semelhantes aos encontrados em Minas Gerais e inferior ao de cinco macrorregiões mineiras (Leste do Sul, Oeste, Sul, Triângulo do Norte e do Sul) para o ano de $2011^{13}$. Contudo, obteve melhor desempenho quando comparado aos valores encontrados para o Brasil e Grandes Regiões, que também ficaram abaixo da meta nacional estabelecida, apresentando valores inferiores a 0,20 nos anos de 2010 e $2011^{28}$.

A princípio, tal achado não parece estar relacionado à disponibilidade de mamógrafos na região. Em 2002, o Ministério da Saúde estabeleceu a cobertura assistencial de mamógrafos no país de um aparelho para 240 mil habitantes ${ }^{30}$. No estado de São Paulo, existiam 1.176 mamógrafos em 2013, incluindo equipamentos da rede SUS e rede privada não conveniada ao SUS, sendo que 433 desses aparelhos eram de uso exclusivo do SUS. Em média, o estado dispunha de 4,4 mamógrafos por 240 mil habitantes, com uma média de utilização do equipamento (capacidade) em torno de $60 \%^{31,32}$. Em 2013, a região da Grande São Paulo, que corresponde à RM estudada, possuía 135 mamógrafos para utilização no SUS, com 3,4 aparelhos para 240.000 usuárias SUS dependentes ${ }^{32}$, superando em torno de duas vezes a cobertura determinada pelo Ministério da Saúde.

O indicador razão de mamografias em mulheres de 50 a 69 anos tem sido utilizado como proxy da cobertura mamográfica, uma vez que mede o número de exames e não o número de mulheres que realizou mamografia ${ }^{26}$. Este contexto ocorre em decorrência da ausência de um registro individualizado por mulher no SISMAMA, limitação que está sendo corrigida pelo SISCAN. Este sistema, que se encontra em fase de implantação, integra as informações do SISMAMA e SISCOLO, registrando cada procedimento realizado pela mulher de forma individualizada ${ }^{33,34}$.

Constatou-se que metade das mulheres avaliadas foram rastreadas dentro da faixa etária preconizada pelas diretrizes nacionais (50-69 anos), no entanto, aproximadamente 35\% pertenciam à faixa etária de 40-49 anos, tanto na RM quanto no IE. Esse percentual de $35 \%$ representou 1.071.784 mamografias de rastreamento realizadas no triênio, que poderiam ter contribuído para o atendimento à demanda da população-alvo. Tal achado pode explicar, em grande parte, a diferença observada entre a produção de exames mamográficos no período e a cobertura da população-alvo do rastreamento. Nos estudos de Correa et al. ${ }^{13}$ e Tomazelli et al. ${ }^{17}$, referentes aos anos de 2010 e 2011, foram encontrados percentuais por faixa etária semelhantes aos do presente estudo para o estado de Minas Gerais e Brasil, respectivamente. Tais achados demonstram a não adequação às recomendações do Ministério da Saúde referentes à faixa etária preconizada ${ }^{35}$, o que pode estar relacionado ao atendimento às orientações das sociedades médicas especializadas, que orientam o rastreamento também na faixa etária de 40-49 anos ${ }^{14}$. Neste sentido, vale ressaltar que a Organização Mundial de Saúde (OMS) ${ }^{28}$ e a United States Preventive Services Task Force (USPSTF) ${ }^{36}$ questionam a eficácia da recomendação 
do rastreamento mamográfico na faixa etária de 40-49 anos, sinalizando para uma relação entre benefícios e danos nesta faixa etária mais desfavorável do que na faixa etária de 50-69 anos. Tal fato pode ser justificado pela maior densidade mamária das mulheres com idade entre 40 e 49 anos, o que reduz a sensibilidade da mamografia, contribuindo para o aumento do número de resultados falso-positivos e gerando excessos na demanda por exames diagnósticos (histopatológicos) e ansiedade nas mulheres sob investigação ${ }^{28,35}$.

Para a faixa etária de 60-69 anos, foi verificado apenas cerca de 18\% de realização de mamografia de rastreamento, independentemente do local avaliado (RM ou IE). Esse resultado pode ser explicado, em grande parte, pela baixa adequação às diretrizes clínicas preconizadas, em especial no que se refere às mulheres mais velhas (a partir de 60 anos) que compõem a população-alvo do rastreamento, assim como pode sinalizar para uma possível queda da adesão ao rastreamento na medida em que aumenta a idade, o que também foi observado em estudo realizado em Taubaté, interior de São Paulo, que registrou taxas decrescentes de adesão ao rastreio mamográfico nas mulheres nesta faixa etária ${ }^{10}$. Existem evidências de que a adesão aos programas de rastreamento do câncer de mama diminui progressivamente segundo a extensão do tempo de acompanhamento ${ }^{37,38}$.

O alto percentual de realização da mamografia com periodicidade anual (cerca de $50 \%$ no triênio), mais proeminente na RM, corrobora com os resultados encontrados no Brasil e em Minas Gerais ${ }^{13,17}$. Por outro lado, o rastreamento bienal para mulheres entre 50-69 é considerado a melhor estratégia disponível atualmente para mulheres com risco médio, uma vez que reduz o percentual de resultados de mamografias falso-positivas e de recomendações de biópsias desnecessárias, contribuindo para reduzir os possíveis danos do rastreamento ${ }^{36,39}$. Tal constatação reforça a necessidade de priorização de ações direcionadas aos grupos de alto risco, especialmente em países com recursos de saúde limitados.

O comportamento observado em relação ao BI-RADS ${ }^{\circledR}$ no estado de São Paulo foi similar aos achados de outros estudos $13,17,27$. Foi observada melhoria nos percentuais dos exames classificados como inconclusivos (BI-RADS ${ }^{\circledR} 0$ ) nas mamografias de rastreamento, alcançando a proporção de $10 \%$, também verificada no programa de rastreamento canadens $\mathrm{e}^{40} \mathrm{e}$ bastante próxima dos padrões internacionais que giram em torno dos $10 \%{ }^{41}$.

As mamografias diagnósticas sofreram importante modificação ao longo do período avaliado, de forma mais marcante na RM, com aumento no tempo de espera para a realização do exame e para a obtenção de seu resultado. Estudos realizados no interior de São Paulo encontraram período de espera para a realização da mamografia com variação de 42 a 60 dias em média ${ }^{42,43}$. Em 2011, também foram registradas reduções no percentual de mamografias diagnósticas com realização em até 30 dias em Minas Gerais, Brasil e todas as grandes regiões, exceto Nordeste ${ }^{13,28}$. Esses resultados apontam deficiências no fluxo de marcação e liberação dos laudos mamográficos, que deveriam priorizar as mamografias diagnósticas, visto que, nessas condições, as mulheres já apresentam sinais e sintomas e deveriam ter o diagnóstico confirmado no menor tempo possível ${ }^{28}$.

A redução da razão entre biópsias e resultados de mamografias $\mathrm{BI}-\mathrm{RADS}^{\circledR} 4$ e 5 observada na RM pode ser devida ao aumento progressivo nas mamografias alteradas (BI-RADS ${ }^{\circledR} 4$ e 5) observado ao longo do triênio, não acompanhado pela oferta de biópsias, que, no mesmo período, sofreram uma redução de $50 \%$ no primeiro ano e de $40 \%$ no segundo ano, quando comparados a 2010 (ano de maior produtividade de biópsias). O atraso na confirmação do diagnóstico motivado pela demora na realização do exame histopatológico tem sido observado em alguns estudos feitos no estado de São Paulo. Estudo realizado no Hospital Estadual Mário Covas, localizado na RM de São Paulo, identificou tempo mediano de 72 dias para biópsia ${ }^{44}$. Em Jundiaí, avaliação feita em ambulatório de um serviço público de Saúde da Mulher constatou tempo mediano de 42 dias para realização da biópsia e de 43,5 dias para confirmação do diagnóstico a partir da data da biópsia ${ }^{42}$. Ademais, os dados encontrados em 2011 e 2012 para o Brasil, levantados pelo INCA, são semelhantes aos encontrados na RM e IE paulista ${ }^{35}$.

O presente estudo apresenta, como limitações, a possibilidade de: existir mais de um registro de procedimento de mamografia e/ou biópsia por mulher no mesmo ano, visto que os registros no sistema são feitos a partir do procedimento realizado; os fragmentos de uma 
mesma biópsia serem registrados como exames diferentes; existir subnotificação da realização do exame histopatológico motivada por problemas durante o envio das bases para o SISMAMA; e ausência de informações histopatológicas e de mamografias, quando estes procedimentos diagnósticos são realizados no sistema privado ou particular de saúde.

De acordo com o Sistema de Informação de Beneficiários da Agência Nacional de Saúde Suplementar (SIB/ANS), a taxa média de cobertura de plano de saúde para mulheres na faixa etária de 50 a 69 anos, no período avaliado, era de $40 \%$, maior percentual encontrado entre os estados brasileiros. Em razão de a rede privada de saúde não disponibilizar dados suficientes para que seja calculada a cobertura do rastreamento oferecido no contexto da saúde suplementar, os dados registram apenas os exames realizados em mulheres que foram atendidas no âmbito do SUS. Se não houvéssemos considerado nos cálculos esse percentual de $40 \%$ das mulheres com cobertura de plano de saúde, as estimativas de cobertura de mamografias poderiam ter apresentado melhores resultados, devendo, portanto, ser interpretados com cautela. Ainda cabe ressaltar que a avaliação refere-se ao estado mais desenvolvido do país e que apresenta a maior concentração de oferta de serviços de saúde, em todos os níveis de atenção, contexto que não está presente na maioria dos demais estados brasileiros.

Os resultados reforçam a extrema relevância que o registro das informações seja realizado de forma mais criteriosa nos sistemas de informações existentes, da disponibilização desses dados para que as avaliações possam ser feitas periodicamente e de investimentos no aprimoramento dessas ferramentas.

A atualização das diretrizes para a detecção precoce do câncer de mama destaca a importância do diagnóstico precoce das mulheres sintomáticas. A partir de uma investigação ágil e de qualidade, é possível garantir o início do tratamento adequado em um curto espaço de tempo, que contribuirá para melhorar a sobrevida das mulheres acometidas pela neoplasia ${ }^{45}$. Em cenários socioeconômicos tão heterogêneos como os existentes no país, a priorização da detecção precoce das mulheres sintomáticas é fundamental para se alcançar o controle do câncer de mama ${ }^{45}$.

A partir dos resultados do presente estudo, conclui-se pela necessidade de adequações no rastreamento mamográfico realizado no estado de São Paulo, e, em particular, no que se refere à faixa etária preconizada e a periodicidade, especialmente na região metropolitana, para que, de fato, as ações de detecção precoce sejam efetivas. Destaca-se a importância da implantação de um rastreamento organizado e do estímulo à utilização do sistema de informação do câncer que possibilite o monitoramento e avaliação das ações de detecção precoce e contribua para o aprimoramento da Política Nacional de Prevenção e Controle do Câncer de Mama.

\section{REFERÊNCIAS}

1. Stewart BW, Wild CP. World Cancer Report 2014. Lyon: International Agency for Research on Cancer; 2014.

2. Torre LA, Siegel RL, Ward EM, Jemal A. Global cancer incidence and mortality rates and trends: an update. Cancer Epidemiol Biomarkers Prev. 2016;25(1):16-27. http://dx.doi.org/10.1158/1055-9965.EPI-15-0578. PMid:26667886.

3. Brasil. Ministério da Saúde. Estimativa 2018: incidência de câncer no Brasi. Rio de Janeiro: INCA; 2017.

4. Azevedo e Silva G, Souza-Júnior PRB, Damacena GN, Szwarcwald CL. Detecção precoce do câncer de mama no Brasil: dados da Pesquisa Nacional de Saúde, 2013. Rev Saude Publica. 2017;51(Supl. 1):14s.

5. Girianelli VR, Gamarra JC, Azevedo e Silva G. Os grandes contrastes na mortalidade por câncer do colo uterino e de mama no Brasil. Rev Saude Publica. 2014;48(3):459-67. http://dx.doi.org/10.1590/S00348910.2014048005214. PMid:25119941.

6. Brasil. Ministério da Saúde. Diretrizes para a detecção precoce do câncer de mama. Rio de Janeiro: INCA; 2015.

7. Warner E. Breast-Cancer Screening. N Engl J Med. 2011;365(11):1025-32. http://dx.doi.org/10.1056/ NEJMcp1101540. PMid:21916640.

8. Netto Rodrigues DC, Freitas-Junior R, Da R, Corrêa S, Peixoto JE, Tomazelli JG, et al. Avaliação do desempenho dos centros de diagnóstico na classificação dos laudos mamográficos em rastreamento oportunista do Sistema Único de Saúde (SUS). Radiol Bras. 2013;46(3):149-55. 
9. Corrêa R, Da Silva F, Hortale VA. Rastreamento do câncer de mama no Brasil: quem, como e por quê? Rev Bras Cancerol. 2012;58(1):67-71.

10. Marchi AA, Gurgel MSC. Adesão ao rastreamento mamográfico oportunístico em serviços de saúde públicos e privados. Rev Bras Ginecol Obstet. 2010;32(4):191-7. http://dx.doi.org/10.1590/S010072032010000400007. PMid:20625688.

11. Brasil. Ministério da Saúde. Portaria Conjunta no 19/SAS, de 3 de julho de 2018. Aprova as Diretrizes Diagnósticas e Terapêuticas do Carcinoma de Mama. Diário Oficial da União, Brasília, 2018; Seção 1. http:// dx.doi.org/10.31331/sece.v1i1.599.

12. World Health Organization. WHO position paper on mammography screening. Geneva: World Health Organization; 2014.

13. Corrêa CSL, Pereira LC, Leite ICG, Fayer VA, Guerra MR, Bustamante-Teixeira MT. Rastreamento do câncer de mama em Minas Gerais: avaliação a partir de dados dos sistemas de informações do Sistema Único de Saúde. Epidemiol Serv Saúde. 2017;26(3):481-92.

14. Urban L, Chala L, Bauab S, Schaefer M, Santos R, Maranhão N, et al. Recomendações do Colégio Brasileiro de Radiologia e Diagnóstico por Imagem, da Sociedade Brasileira de Mastologia e da Federação Brasileira das Associações de Ginecologia e Obstetrícia para o rastreamento do câncer de mama. Radiol Bras. 2017;50(4):244-9. http://dx.doi.org/10.1590/0100-3984.2017-0069. PMid:28894332.

15. Brasil. Ministério da Saúde. Portaria n².439/GM, de 8 de dezembro de 2005. Institui a Política Nacional de Atenção Oncológica: promoção, prevenção, diagnóstico, reabilitação e cuidados paliativos, a ser implantada em todas as Unidades Federadas, respeitadas as competências das três. Diário Oficial da União, Brasília, 9 de dezembro de 2005; Seção 1, p. 80-81.

16. Brasil. Ministério da Saúde. Portaria no 779, de 31 de dezembro de 2008. Institui o Sistema de Informação do Controle do Câncer de Mama (SISMAMA) no âmbito do Sistema ùnico de Saúde (SUS). Diário Oficial da União [Internet], Brasília, DF, 2 de janeiro de 2009; Seção 1. p. 38 [citado em 2018 Mar 6]. Disponível em: http://bvsms.saude.gov.br/bvs/saudelegis/sas/2008/prt0779_31_12_2008.html

17. Tomazelli JG, Migowski A, Ribeiro CM, Assis M, Abreu DM, Tomazelli JG, et al. De Assis, De Abreu DMF. Avaliação das ações de detecção precoce do câncer de mama no Brasil por meio de indicadores de processo: estudo descritivo com dados do Sismama, 2010-2011. Epidemiol Serv Saude. 2017;26(1):61-70. http://dx.doi.org/10.5123/S1679-49742017000100007. PMid:27901188.

18. Brasil. Ministério da Saúde. Plano de Ações Estratégicas para o Enfrentamento das Doenças Crônicas Não Transmissíveis (DCNT) no Brasil-2011 a 2022 [Internet]. Brasília: Ministério da Saúde; 2011 [citado em 2018 mar 15]. Disponível em: http://bvsms.saude.gov.br/bvs/publicacoes/plano_acoes_enfrent_dent_2011. pdf

19. Brasil. Ministério da Saúde. Informativo detecção precoce: monitoramento das ações de controle dos cânceres do colo do útero e de mama. INCA. Bol. 2014;5(1):1-8.

20. Instituto Brasileiro de Geografia e Estatística. Brasil em Síntese [Internet]. Rio de Janeiro: IBGE; 2019 [citado em 2018 fev 26]. Disponível em: https://cidades.ibge.gov.br/brasil/sp/panorama

21. Brasil. Ministério da Saúde. Informações de Saúde (TABNET) - Demográficas e Socioeconômicas [Internet] 2017 [citado em 2017 out 16]. Disponível em: http://tabnet.datasus.gov.br/cgi/tabcgi.exe?ibge/cnv/ popsp.def

22. São Paulo. São Paulo: potência em história, economia, cultura e turismo [Internet]. 2018 [citado em 2018 fev 26]. Disponível em: http://www.saopaulo.sp.gov.br/conhecasp/

23. São Paulo. Empresa Paulista de Planejamento Metropolitano AS. Região Metropolitana de São Paulo [Internet]. São Paulo: Emplasa; 2018 [citado em 2018 fev 26]. Disponível em: https://www.emplasa.sp.gov. br/RMSP

24. São Paulo. Lei complementar no 1.139, de 16 de junho de 2011. Reorganiza a Região Metropolitana da Grande São Paulo, cria o respectivo Conselho de Desenvolvimento e dá providências correlatas. Diário Oficial [do] Estado de São Paulo [Internet], São Paulo, 17 de junho de 2011; Executivo. p. 1 [citado em 2018 fev 26]. Disponível em: https://www.al.sp.gov.br/repositorio/legislacao/lei.complementar/2011/lei. complementar-1139-16.06.2011.html

25. São Paulo. Caracterização da assistência oncológica nas Redes Regionais de Atenção à Saúde no estado de São Paulo. São Paulo: Fundação Oncocentro de São Paulo; 2014. p. 1-6.

26. Brasil. Ministério da Saúde. Ficha técnica de indicadores relativos às ações de controle do câncer de mama. Rio de Janeiro: Instituto Nacional de Câncer José de Alencar Gomes da Silva; 2014.

27. Azevedo Silva CG, Bustamante-Teixeira MT, Aquino EML, Tomazelli JG, Dos-Santos-Silva I. Acesso à detecção precoce do câncer de mama no Sistema Único de Saúde: uma análise a partir dos dados do Sistema de Informações em Saúde. Cad Saude Publica. 2014;30(7):1537-50. http://dx.doi.org/10.1590/0102311X00156513. PMid:25166949. 
28. Brasil. Ministério da Saúde. Informativo detecção precoce: monitoramento das ações de controle dos cânceres do colo do útero e de mama. Bol. 2012;3(3):1-10.

29. São Paulo. Plano de Ação Regional de Prevenção e Controle do Câncer da Região Rota dos Bandeirantes. São Paulo; 2014. 63 p.

30. Brasil. Ministério da Saúde. Portaria no 1101/GM, de 12 de junho de 2002. Estabelece parâmetros de cobertura assistencial no âmbito do Sistema Único de Saúde - SUS. Brasília; 2002.

31. Xavier DR, Oliveira RAD, Matos VP, Viacava F, Carvalho CC. Cobertura de mamografias, alocação e uso de equipamentos nas Regiões de Saúde. Saúde Debate. 2016;40(110):20-35. http://dx.doi.org/10.1590/01031104201611002.

32. Mendes JDV, Cecílio MAM, Osiano VLRL. Produção de Mamografias no SUS do Estado de São Paulo. BEPA. 2013;10(120):17-23.

33. Brasil. Ministério da Saúde. Portaria no 3.394, de 30 de dezembro de 2013. Institui o Sistema de Informação de Câncer (SICAN) no âmbito do Sistema ùnico de Saúde (SUS). Diário Oficial da União [Internet], Brasília, DF, de 30 de dezembro de 2013 [acesso 2018 Mar 6]. Disponível em: http://bvsms.saude.gov.br/bvs/ saudelegis/gm/2013/prt3394_30_12_2013.html

34. Brasil. Ministério da Saúde. Sistema de informação do câncer: manual preliminar para apoio à implantação. Rio de Janeiro: INCA; 2013. 143 p.

35. Brasil. Ministério da Saúde. Informativo detecção precoce: monitoramento das ações de controle dos cânceres do colo do útero e mama. INCA. Bol. 2013;4(3):1-7.

36. Siu AL, U.S. Preventive Services Task Force. Screening for breast cancer: U.S. Preventive services task force recommendation statement. Ann Intern Med. 2016;164(4):279-96. http://dx.doi.org/10.7326/M15-2886. PMid:26757170.

37. Clark MA, Rakowski W, Bonacore LB. Repeat mammography: prevalence estimates and considerations for assessment. Ann Behav Med. 2003;26(3):201-11. http://dx.doi.org/10.1207/S15324796ABM2603_05. PMid:14644696.

38. Boudreau DM, Luce CL, Ludman E, Bonomi AE, Fishman PA. Concordance of population-based estimates of mammography screening. Prev Med. 2007;45(4):262-6. http://dx.doi.org/10.1016/j.ypmed.2007.07.011. PMid:17698182.

39. Mandelblatt JS, Stout NK, Schechter CB, Van Den Broek JJ, Miglioretti DL, Krapcho M, et al. Collaborative modeling of the benefits and harms associated with different U.S. Breast cancer screening strategies. Ann Intern Med. 2016;164(4):215-25. http://dx.doi.org/10.7326/M15-1536. PMid:26756606.

40. Public Health Agency of Canada (CA). Organized breast cancer screening programs in Canada: report on program performance in 2001 and 2006. Ottawa: Public Health Agency of Canada; 2011.

41. Brasil. Ministério da Saúde. Informativo detecção precoce: monitoramento das ações de controle dos cânceres do colo do útero e de mama. INCA. Bol. 2013;4:1-9.

42. Traldi MC, Galvão P, Morais SS, Fonseca MRCC. Demora no diagnóstico de câncer de mama de mulheres atendidas no Sistema Público de Saúde. Cad Saude Colet. 2016;24(2):185-91. http://dx.doi. org/10.1590/1414-462X201600020026.

43. Maia EMC, Maia LB, Valente FM, Machado RB, Borges JBR. Tempo decorrido entre a primeira consulta e o tratamento definitivo nos caos de câncer de mama no Sistema de Saúde público da cidade de Jundiaí. Rev Bras Mastol. 2006;16(1):23-6.

44. Trufelli DC, Miranda VC, Dos Santos MBB, Fraile NMP, Pecoroni PG, Gonzaga SFR, et al. Análise do atraso no diagnóstico e tratamento do câncer de mama em um hospital público. Rev Assoc Med Bras. 2008;54(1):726. http://dx.doi.org/10.1590/S0104-42302008000100024. PMid:18392490.

45. Dos-Santos-Silva I. Políticas de controle do câncer de mama no Brasil: quais são os próximos passos? Cad Saude Publica. 2018;34(6):e00097018. http://dx.doi.org/10.1590/0102-311x00097018. PMid:29947658. 